\title{
The Kaon Semileptonic Form Factor from Domain Wall QCD at the Physical Point
}

\section{Jung}

Brookhaven National Laboratory, Upton, NY 11973, USA

E-mail: chulwoo@physics.columbia.edu

\author{
N.H. Christ, R.D. Mawhinney, D.J. Murphy*, H. Yin \\ Department of Physics, Columbia University, New York, NY 10027, USA \\ E-mail: nhc@phys.columbia.edu, rdmephysics.columbia.edu, \\ dmurphyephys.columbia.edu, yinnhtephys.columbia.edu
}

\section{P.A. Boyle}

School of Physics and Astronomy, University of Edinburgh, EH9 3JZ, UK

E-mail: paboyle@ph.ed.ac.uk

\section{N. Garron}

School of Computing and Mathematics and Centre for Mathematical Science, Plymouth

University, Plymouth PL4 8AA, UK

E-mail: ngarroneph.ed.ac.uk

\section{J.M. Flynn, A. Jüttner, C.T. Sachrajda, F. Sanfilippo}

School of Physics and Astronomy, University of Southampton, Southampton SO17 1BJ, UK

E-mail: jflynn@phys.soton.ac.uk, juettner@soton.ac.uk,

ctsesoton.ac.uk, f.sanfilippodsoton.ac.uk

We present the first calculation of the kaon semileptonic form factor at zero momentum transfer in $N_{f}=2+1$ domain wall QCD with physical quark masses. We jointly analyze two sets of lattice simulations with different lattice spacings and large physical volumes. We obtain the continuum result $f_{+}^{K \pi}(0)=0.9685(34)_{\text {statistical }}(14)_{\text {systematic }}$. Combining this calculation with data from experimental measurements of $K \rightarrow \pi$ decays, we predict the CKM matrix element $\left|V_{u s}\right|=0.2233(5)_{\text {experiment }}(9)_{\text {lattice }}$.

The 32nd International Symposium on Lattice Field Theory,

23-28 June, 2014

Columbia University New York, NY

\footnotetext{
* Speaker.

${ }^{\dagger}$ Current address: 1600 Ampitheatre Parkway, Mountain View, CA 94043.
} 


\section{Introduction}

In the Standard Model of particle physics flavor-changing weak decays are parametrized by the $3 \times 3$, unitary Cabibbo-Kobayashi-Maskawa $(\mathrm{CKM})$ matrix [1,2]. The elements of the CKM matrix, $V_{i j}$, parametrize decays of a quark with flavor $i$ to a quark with flavor $j$. Accurate determinations of the CKM matrix elements are necessary to fix four of the free parameters of the Standard Model, as well as to understand CP violation in weak decays and to test unitarity ${ }^{1}$. Precision measurements of the $K \rightarrow \pi l v\left(K_{l 3}\right)$ decay rate, together with lattice QCD calculations of the non-perturbative $K_{l 3}$ form factor $f_{+}^{K \pi}(0)$, give the most precise constraint on $\left|V_{u s}\right|$ to date. In this talk we report on the RBC/UKQCD collaboration's most recent calculation of $f_{+}^{K \pi}(0)$, which incorporates two new physical pion mass Möbius domain wall fermion ensembles, as well as its implications for the value of $\left|V_{u s}\right|$ and Standard Model unitarity.

\section{Measurement Strategy}

Experimental measurements of the $K^{0} \rightarrow \pi^{-}$decay rate constrain the product $\left|V_{u s}\right| f_{+}^{K \pi}(0)=0.2163(5)$ [3]. To extract $\left|V_{u s}\right|$ we compute the QCD matrix element

$$
\left\langle\pi\left(p_{\pi}\right)\left|V_{\mu}\right| K\left(p_{K}\right)\right\rangle=f_{+}^{K \pi}\left(q^{2}\right)\left(p_{K}+p_{\pi}\right)_{\mu}+f_{-}^{K \pi}\left(q^{2}\right)\left(p_{K}-p_{\pi}\right)_{\mu},
$$

where $V_{\mu}=\bar{u} \gamma_{\mu} s$ is the flavor-changing vector current, and $q=p_{K}-p_{\pi}$ is the momentum transfer between the kaon and pion. One can also relate the form factor to a similar matrix element of the scalar density $S=\bar{u} s$ using a Ward identity for the vector current. At the kinematical point $q^{2}=0$ the analogue of eqn. (2.1) for the scalar density is

$$
\left.\left\langle\pi\left(p_{\pi}\right)|S| K\left(p_{K}\right)\right\rangle\right|_{q^{2}=0}=\frac{m_{K}^{2}-m_{\pi}^{2}}{m_{s}-m_{l}} f_{+}^{K \pi}(0),
$$

where $m_{u}=m_{d} \equiv m_{l}$ is the (degenerate) light quark mass. In all of our calculations we consider a kaon at rest and a pion with momentum $\vec{p}_{\pi}$, where twisted boundary conditions [10] are used to tune $\vec{p}_{\pi}$ such that $q^{2}=0$. We also make use of the all-mode averaging (AMA) technique as described in [5, 14].

\subsection{Simulation Parameters}

We simulate $N_{f}=2+1$ domain wall QCD using a series of ensembles with unitary pion masses ranging from $693 \mathrm{MeV}$ down to the physical value of $139 \mathrm{MeV}$. Our older, unphysical ensemble sets $A$ and $C$ were generated using the Shamir kernel $[6,7]$ and were analyzed in [4]. We have generated two new physical pion mass Möbius domain wall fermion [8] ensembles, denoted $A_{\text {phys }}$ and $C_{\text {phys }}$, with large volumes, which are the focus of this analysis, and are discussed in detail in [5]. All ensembles use the Iwasaki gauge action [9]. The full set of ensembles is summarized in table 1.

\footnotetext{
${ }^{1}$ In particular, tension between the experimentally determined values of the CKM matrix elements and unitarity may prove to be an important indicator of new physics.
} 


\begin{tabular}{c||cccccccccc} 
Ensemble & Action & $\beta$ & $a(\mathrm{fm})$ & $L / a$ & $T / a$ & $a m_{l}$ & $a m_{s}^{\text {sea }}$ & $a m_{s}^{\text {val }}$ & $m_{\pi}(\mathrm{MeV})$ & $m_{\pi} L$ \\
\hline \hline$A_{3}$ & DWF+I & 2.13 & 0.11 & 24 & 64 & 0.03 & 0.04 & 0.04 & 693 & 9.3 \\
$A_{2}$ & DWF+I & 2.13 & 0.11 & 24 & 64 & 0.02 & 0.04 & 0.04 & 575 & 7.7 \\
$A_{1}$ & DWF+I & 2.13 & 0.11 & 24 & 64 & 0.01 & 0.04 & 0.04 & 431 & 5.8 \\
$A_{5}^{4}$ & DWF+I & 2.13 & 0.11 & 24 & 64 & 0.005 & 0.04 & 0.04 & 341 & 4.6 \\
$A_{5}^{3}$ & DWF+I & 2.13 & 0.11 & 24 & 64 & 0.005 & 0.04 & 0.03 & 341 & 4.6 \\
\hline$C_{8}$ & DWF+I & 2.25 & 0.08 & 32 & 64 & 0.008 & 0.03 & 0.025 & 431 & 5.5 \\
$C_{6}$ & DWF+I & 2.25 & 0.08 & 32 & 64 & 0.006 & 0.03 & 0.025 & 360 & 4.8 \\
$C_{4}$ & DWF+I & 2.25 & 0.08 & 32 & 64 & 0.004 & 0.03 & 0.025 & 304 & 4.1 \\
\hline$A_{\text {phys }}$ & MDWF+I & 2.13 & 0.11 & 48 & 96 & 0.00078 & 0.0362 & 0.0362 & 139 & 3.8 \\
\hline$C_{\text {phys }}$ & MDWF+I & 2.25 & 0.08 & 64 & 128 & 0.000678 & 0.02661 & 0.02661 & 139 & 3.9
\end{tabular}

Table 1: Summary of ensembles used in this analysis. DWF and MDWF denote domain wall fermions with the Shamir and Möbius kernels, respectively, and I denotes the Iwasaki gauge action. $L$ and $T$ are the size of the lattice in the spatial and temporal directions, respectively. $m_{\pi}$ is the unitary pion mass.

\section{Data Analysis}

For additional detail regarding the data analysis we refer the reader to our accompanying publication [14]. In this talk we will merely paraphrase the final results.

\subsection{Fits on the Physical Point Ensembles}

We extract the $K_{l 3}$ form factor from suitably constructed ratios of Euclidean three-point functions [10]:

$$
R_{V}^{(\mu)}\left(\Delta_{K \pi}, t\right)=2 \sqrt{E_{\pi} m_{K}}\left[\frac{\mathscr{C}_{K \pi}^{(\mu)}\left(t, \Delta_{K \pi}, \vec{p}_{\pi}\right) \mathscr{C}_{\pi K}^{(\mu)}\left(t, \Delta_{K \pi}, \vec{p}_{\pi}\right)}{\widetilde{\mathscr{C}}_{\pi}\left(\Delta_{K \pi}\right) \mathscr{\mathscr { C }}_{K}\left(\Delta_{K \pi}\right)}\right]^{1 / 2} \simeq \frac{1}{Z_{V}}\left(\left(p_{K}+p_{\pi}\right)^{\mu} f_{+}^{K \pi}(0)+\left(p_{K}-p_{\pi}\right)^{\mu} f_{-}^{K \pi}(0)\right)
$$

for the vector current, and

$$
R_{S}\left(\Delta_{K \pi}, t\right)=2 \sqrt{E_{\pi} m_{K}}\left(\frac{m_{s}-m_{l}}{m_{K}^{2}-m_{\pi}^{2}}\right)\left[\frac{\mathscr{C}_{K \pi}^{(S)}\left(t, \Delta_{K \pi}, \vec{p}_{\pi}\right) \mathscr{C}_{\pi K}^{(S)}\left(t, \Delta_{K \pi}, \vec{p}_{\pi}\right)}{\widetilde{\mathscr{C}}_{\pi}\left(\Delta_{K \pi}\right) \widetilde{\mathscr{C}}_{K}\left(\Delta_{K \pi}\right)}\right]^{1 / 2} \simeq f_{+}^{K \pi}(0)
$$

for the scalar density. These ratios are equal to the right hand side up to noise and excited state contamination for large $K-\pi$ separations $\Delta_{K \pi} \gg 1$ and $t$ far from the source and sink. Here $\mathscr{C}_{K \pi}^{(\mu)}$ and $\mathscr{C}_{K \pi}^{(S)}$ are the vector and scalar three-point functions defined by the matrix elements 2.1 and 2.2, respectively, where $t$ denotes the time of the operator insertion, $\Delta_{K \pi} \equiv\left|t_{K}-t_{\pi}\right|$ is the $K-\pi$ separation, and $Z_{V}$ is the vector current renormalization factor. $\mathscr{C}_{\pi K}^{(\mu)}$ and $\mathscr{C}_{\pi K}^{(S)}$ are the corresponding threepoint functions for the time-reversed process $\pi \rightarrow K . \widetilde{\mathscr{C}}_{\pi, K}(t) \equiv \mathscr{C}_{\pi, K}(t)-\frac{1}{2} \mathscr{C}_{\pi, K}(T / 2) e^{-E_{\pi, K}(T / 2-t)}$ denotes the pion/kaon two-point function with the backward propagating around-the-world mode analytically removed using the fitted pion/kaon energy $E_{\pi, K}$. The vector current renormalization factor $Z_{V}$ is extracted from a similar analysis: we compute the analogue of 2.1 for the electromagnetic current and two pions or two kaons at rest, and fit to the ratios

$$
R_{Z}^{\pi}\left(\Delta_{\pi \pi}, t\right)=\frac{\widetilde{\mathscr{C}}_{\pi}\left(\Delta_{\pi \pi}\right)}{\mathscr{C}_{\pi \pi}^{(0)}\left(t, \Delta_{\pi \pi}, \overrightarrow{0}\right)} \simeq Z_{V}^{\pi}, \quad R_{Z}^{K}\left(\Delta_{K K}, t\right)=\frac{\widetilde{\mathscr{C}}_{K}\left(\Delta_{K K}\right)}{\mathscr{C}_{K K}^{(0)}\left(t, \Delta_{K K}, \overrightarrow{0}\right)} \simeq Z_{V}^{K} .
$$


In this case the form factors are trivial - current conservation implies $f_{+}^{\pi \pi}(0)=f_{+}^{K K}(0)=1$ and $f_{-}^{\pi \pi}(0)=f_{-}^{K K}(0)=0-$ allowing us to extract $Z_{V}$.

For the fit to the scalar ratio 3.2 we observe a non-negligible sensitivity to the choice of fit range, presumably due to contamination from excited states. This is most pronounced on the $C_{\text {phys }}$ ensemble, for which the plateau exhibits a small but clear upward slope. To account for this, we fit a model with includes an exponentially decaying excited state

$$
f_{+}^{K \pi}\left(0, \Delta_{K \pi}\right)=f_{+}^{K \pi}(0)+C e^{-m \Delta_{K} \pi}
$$

We find that this works well in practice: the value of $f_{+}^{K \pi}(0)$ from this exponential fit is consistent with the value we get from fitting a constant, but is stable under variations of the fit range. Example fits from the $C_{\mathrm{phys}}$ ensemble are shown in figure 1.
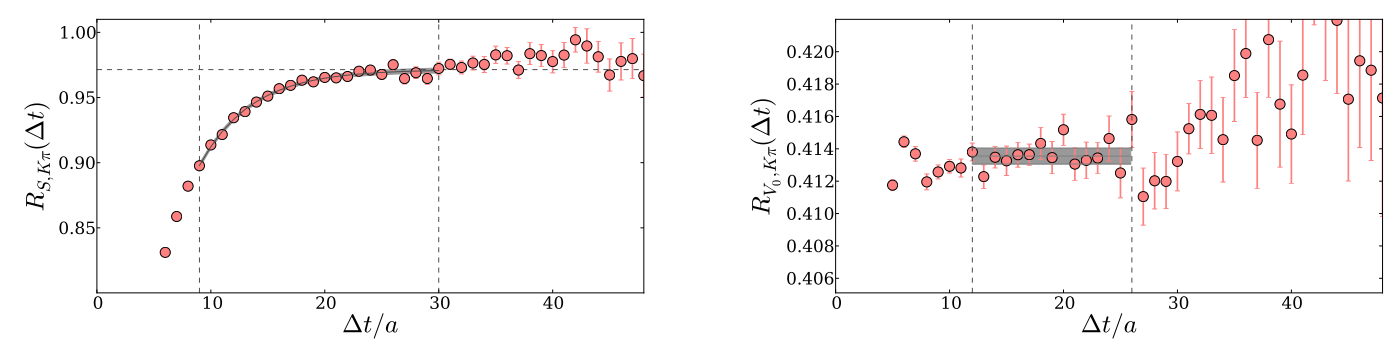

Figure 1: Fits to extract $f_{+}^{K \pi}(0)$ through the scalar density (left), and through the temporal component of the vector current (right) on the $C_{\text {phys }}$ ensemble. Here $\Delta t$ is the $K-\pi$ separation: for even values of $\Delta t$ we evaluate the ratio $R$ at the midpoint $\Delta t / 2$, and for odd values of $\Delta t$ we average the two values of $R$ straddling the midpoint, which does not lie on the lattice.

\subsection{Corrections to the Physical Point}

The values for the twist angles and input quark masses of the physical point ensembles were based on estimates of the spectrum computed from a small number of configurations early in the data generation run. As a result, there is a small discrepancy between the simulated $q^{2}$ and spectrum and the true physical point. We emphasize, however, that this discrepancy is small and the corrections described here are smaller than the statistical errors we quote. In this section we discuss our method for performing this correction independently on the two ensemble sets $A$ and $C$, resulting in two values of $f_{+}^{K \pi}(0)$ at different (finite) lattice spacings. We then perform a continuum extrapolation, which is described in the following section.

To correct the momentum transfer to $q^{2}=0$ we fit a pole ansatz $f_{+}^{K \pi}\left(q^{2}\right)=f_{+}^{K \pi}(0) /(1+$ $\left.q^{2} / M^{2}\right)$ to $f_{+}^{K \pi}\left(q^{2}\right)$ computed at two different kinematical points: the value of $q^{2}$ corresponding to our choice of twist angle, and $q_{\max }^{2}=\left(m_{K}-m_{\pi}\right)^{2}$. We then determine $f_{+}^{K \pi}(0)$ from the fit, and use this corrected value for the form factor in all subsequent steps of the analysis. We have also checked that if we instead use a linear ansatz the change in the result is negligible.

Likewise, we must make a small correction to $f_{+}^{K \pi}(0)$ from the simulated values of $m_{\pi}$ and $m_{K}$ to the physical values $m_{\pi^{-}}=139.6 \mathrm{MeV}$ and $m_{K^{0}}=497.6 \mathrm{MeV}$ [12]. We have considered a 
number of ansätze for performing this correction, which we divide into families $\mathscr{A}, \mathscr{B}, \mathscr{E}$, and $\mathscr{F}$ :

$$
\left\{\begin{array}{l}
\text { fit } \mathscr{A}: f_{+}^{K \pi}\left(q^{2}=0, m_{\pi}^{2}, m_{K}^{2}\right)=1+f_{2}\left(f, m_{\pi}^{2}, m_{K}^{2}\right) \\
\text { fit } \mathscr{B}: f_{+}^{K \pi}\left(q^{2}=0, m_{\pi}^{2}, m_{K}^{2}\right)=1+f_{2}\left(f, m_{\pi}^{2}, m_{K}^{2}\right)+A_{1}\left(m_{K}^{2}+m_{\pi}^{2}\right)\left(m_{K}^{2}-m_{\pi}^{2}\right)^{2} \\
\text { fit } \mathscr{E}: f_{+}^{K \pi}\left(q^{2}=0, m_{\pi}^{2}, m_{K}^{2}\right)=A+A_{0} \Delta M^{2} \\
\text { fit } \mathscr{F}: f_{+}^{K \pi}\left(q^{2}=0, m_{\pi}^{2}, m_{K}^{2}\right)=A+A_{0} \Delta M^{2}+A_{1}\left(m_{K}^{2}+m_{\pi}^{2}\right) \Delta M^{2}
\end{array}\right.
$$

Here $\Delta M^{2} \equiv\left(m_{K}^{2}-m_{\pi}^{2}\right)^{2} / m_{K}^{2}$, and $f_{2}$ is the NLO term from $S U(3)$ chiral perturbation theory $(\chi \mathrm{PT})$. The full expression for $f_{2}$ can be found in [4], where we have previously studied these ansätze. We have also explored several different cuts for the heaviest simulated $m_{\pi}$ included in the fits. Here we will only paraphrase the results: the details of all of the fits we have performed can be found in [14]. We find that even with a mass cut the fits using NLO $\chi \mathrm{PT}$ are of poor quality, and thus we discard fits $\mathscr{A}$ and $\mathscr{B}$ from the final analysis, in favor of fits $\mathscr{E}$ and $\mathscr{F}$, where $f_{2}$ has been replaced by its Taylor expansion ${ }^{2}$ in $m_{K}^{2}-m_{\pi}^{2}$. Fits $\mathscr{E}$ and $\mathscr{F}$ are both of good quality, however, we lack sufficient data to constrain fit $\mathscr{F}$ for $f_{+}^{K \pi}(0)$ computed through the scalar density, so we choose fit $\mathscr{E}$ for our final result. We again emphasize that this interpolation should be thought of as a small correction to mistuning in $A_{\text {phys }}$ and $C_{\text {phys }}$, as one can see from figure 2.

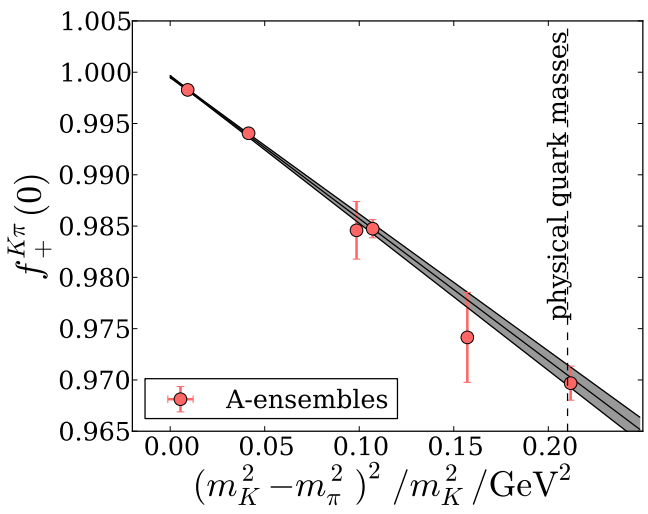

(a) Fit $\mathscr{E}$, no mass cut

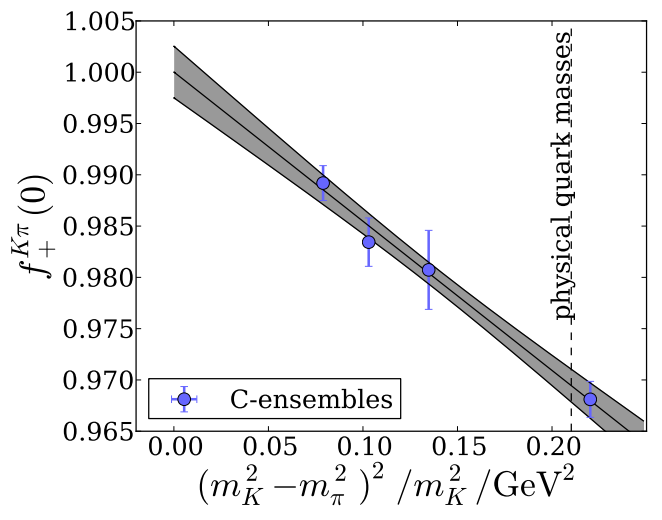

(b) Fit $\mathscr{E}$, no mass cut

Figure 2: Examples of the interpolation to physical mass.

\subsection{Continuum Extrapolation}

After performing the corrections described in section 3.2 we are left with three values of $f_{+}^{K \pi}(0)$ - two independent normalizations of the vector current matrix element 3.1 using $Z_{V}^{\pi}$ and $Z_{V}^{K}$, as well as the scalar matrix element 3.2 - at each of the two lattice spacings considered in our analysis. While we could perform three independent linear extrapolations to the continuum, we instead chose to impose universality, and extrapolate all three calculations of the form factor to a common continuum limit. This is depicted in figure 3. Repeating the full analysis with different choices of the mass cut does not change the result within the statistical error: we choose $m_{\pi} \leq$

\footnotetext{
${ }^{2}$ Another possibility is to fit to NNLO $\chi \mathrm{PT}$, however, we have too little data to constrain such a fit.
} 
$450 \mathrm{MeV}$ as a reasonable mass cut to quote a final result, for which we obtain $f_{+}^{K \pi}(0)=0.9685(34)$ in the continuum, where the error is purely statistical, and includes the uncertainty in the lattice spacings for the $A$ and $C$ ensembles.

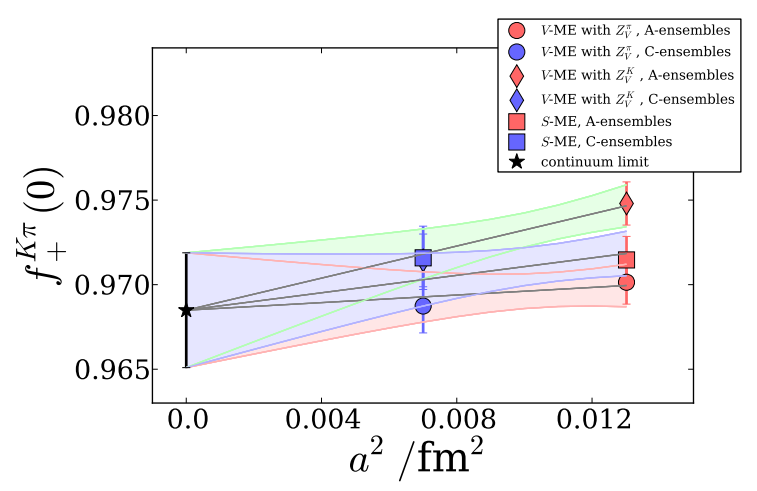

Figure 3: Joint extrapolation to a common continuum limit.

\subsection{Final Result and Error Budget}

After interpolating in $q^{2}, m_{\pi}$, and $m_{K}$ to the physical point, and extrapolating to the continuum, we are left with the following systematic errors:

- Finite Volume $(F V)$ : Since the $K \rightarrow \pi$ matrix element contains only single particle initial and final states, we expected finite volume effects to be exponentially suppressed in $m_{\pi} L$. We naively estimate these effects to be of order $\left(1-f_{+}^{K \pi}(0)\right) e^{-m_{\pi} L}=0.0007$. ChPT [13] estimates an error approximately twice as large for the $A_{\text {phys }}$ and $C_{\text {phys }}$ ensembles. Thus, we quote twice our naive error -0.0014 - as our value for the FV systematic.

- Partial Quenching: The calculations on ensemble $A_{5}^{3}$ and the full $C$ ensemble set were performed with a partially quenched strange quark. We expect any associated systematic errors to be small compared to the other errors we quote, and we have explicitly checked that excluding ensemble $A_{5}^{3}$ from the analysis does not change the result.

- Isospin Breaking: The unitary light quarks in our study are isospin symmetric, unlike the physical up and down quarks. We again expect this to be negligible compared to statistical and FV errors.

Taking these into account, we obtain our final result

$$
f_{+}^{K \pi}(0)=0.9685(34)_{\mathrm{stat}}(14)_{\mathrm{FV}}
$$

leading to the prediction

$$
\left|V_{u s}\right|=0.2233(5)_{\text {experiment }}(9)_{\text {lattice }} \text {. }
$$

Together with $\left|V_{u d}\right|=0.97425$ (22) from super-allowed nuclear $\beta$-decay measurements [12], and neglecting $\left|V_{u b}\right| \approx 10^{-3}$, we observe a $\sim 1.5 \sigma$ tension with first-row CKM unitarity

$$
1-\left|V_{u d}\right|^{2}-\left|V_{u s}\right|^{2}=0.0010(4)_{V_{u d}}(2)_{V_{u s}} \exp _{V_{u s s}^{\text {lat }}}=0.0010(6) .
$$




\section{Conclusion}

In this talk we have presented a first-principles calculation of the kaon semileptonic form factor with vanishing momentum transfer and physical light quark masses in domain wall QCD. We have also demonstrated how to utilize our older, heavy pion mass ensembles to correct for slight mistunings and extrapolate to the continuum. We find that finite volume errors are now the dominant source of systematic error. In the future we hope to explicitly include electromagnetic and isospin breaking effects in our calculations.

\section{References}

[1] N. Cabibbo, Unitary Symmetry and Leptonic Decays, Phys.Rev.Lett. 10 (1963) 531-533.

[2] M. Kobayashi and T. Maskawa, CP Violation in the Renormalizable Theory of Weak Interaction, Prog.Theor.Phys. 49 (1973) 652-657.

[3] M. Antonelli et al., An Evaluation of $\left|V_{u s}\right|$ and Precise Tests of the Standard Model from World Data on Leptonic and Semileptonic Kaon Decays, Eur.Phys.J. C69 (2010) 399-424, [arXiv: 1005.2323$]$.

[4] P.A. Boyle et al., The Kaon Semileptonic Form Factor with Near Physical Domain Wall Quarks, JHEP 1308 (2013) 132, [arXiv: 1305 . 7217].

[5] T. Blum et al., Domain Wall QCD with Physical Quark Masses, [arXiv: 1411.7017$].$

[6] D.B. Kaplan, A Method for Simulating Chiral Fermions on the Lattice, Phys.Lett. B288 (1992) 342-347, [arXiv: hep-lat/9206013].

[7] Y. Shamir, Chiral Fermions from Lattice Boundaries, Nucl.Phys. B406 (1993) 90-106, [arXiv:hep-lat/9303005].

[8] R.C. Brower, H. Neff, and K. Orginos, The Möbius Domain Wall Fermion Algorithm, [arXiv:1206.5214].

[9] Y. Iwasaki and T. Yoshie, Renormalization Group Improved Action for SU(3) Lattice Gauge Theory and the String Tension, Phys.Lett. B143 (1984) 449.

[10] P.A. Boyle et al., Hadronic Form Factors in Lattice QCD at Small and Vanishing Momentum Transfer, JHEP 05 (2007) 016, [arXiv: hep-lat / 0703005 ].

[11] T. Blum, T. Izubuchi, and E. Shintani, A New Class of Variance Reduction Techniques Using Lattice Symmetries, [arXiv:1208.4349].

[12] Particle Data Group, K. Olive et al., Review of Particle Physics, Chin.Phys. C38 (2014) 090001.

[13] K. Ghorbani and H. Ghorbani, Kaon Semi-Leptonic Form Factor at Zero Momentum Transfer in Finite Volume, Eur.Phys.J. A49 (2013) 134, [arXiv:1301.0919].

[14] P.A. Boyle et al., The Kaon Semileptonic Form Factor in $N_{f}=2+1$ Domain Wall Lattice QCD with Physical Light Quark Masses, [arXiv: 1504.01692$].$ 